\title{
Microwave dielectric properties of nanostructured nickel ferrite
}

\author{
JOHN JACOB, M ABDUL KHADAR*, ANIL LONAPPAN ${ }^{\dagger}$ and $K$ T MATHEW ${ }^{\dagger}$ \\ Department of Physics, ${ }^{\dagger}$ Also at Centre for Nanoscience and Nanotechnology, University of Kerala, \\ Kariavattom Campus, Thiruvananthapuram 695 581, India \\ ${ }^{\dagger}$ Department of Electronics, Cochin University of Science and Technology, Kochi 682 022, India
}

MS received 15 February 2008; revised 8 July 2008

\begin{abstract}
Nickel ferrite is one of the important ferrites used in microwave devices. In the present work, we have synthesized nanoparticles of nickel ferrite using chemical precipitation technique. The crystal structure and grain size of the particles are studied using XRD. The microwave dielectric properties of nanostructured nickel ferrite samples of three different average grain sizes and those of two sintered samples were studied. The parameters like dielectric constant, dielectric loss and heating coefficient of the nanoparticles samples are studied in the frequency range from 2.4 to $4 \mathrm{GHz}$. The values of these parameters are compared with those of sintered pellets of the same samples. All these parameters show size dependent variations.
\end{abstract}

Keywords. Nickel ferrite; nanostructured; microwave dielectric; co-precipitation.

\section{Introduction}

Ferrites find wide technological applications in making cores of audio frequency and high frequency transformers, coils (inductors), chokes, permanent magnets, magnetooptical displays, microwave absorbers, wave guides in the $\mathrm{GHz}$ region and chlorine gas sensors (Gotic et al 1998; Gopal Reddy et al 1999).

Among the ferrites, the spinel type ferrites are particularly important since they can be used as electromagnetic wave absorbers or electromagnetic interference (EMI) suppressors in the $\mathrm{MHz}$ frequency range like the TV ghost suppressor (Green et al 1964; Goldman 1990; Nakamura et al 1994; Han et al 1995; Luo et al 2000; Meshram et al 2002). Ferrites are also used in camouflaging military aircrafts and missiles against radar detection (Meshram et al 2002). Among the spinel type ferrites, nickel ferrite is a suitable material for microwave applications (Nicolas 1980). It is noted for its high Curie temperature and good temperature stability of saturation magnetization (Vladikova et al 1989).

The dielectric properties of nickel ferrite could be varied by the addition of small traces of cobalt, manganese and copper, and also by bringing deviation from stoichiometry (Van Uitert 1956a,b; Malinofski and Babbitt 1964; Hudson 1970). Microwave behaviour of nickel ferrite prepared by sol-gel method was reported by Giannakopoulou et al (2002). As far as the authors know, such studies on size dependent microwave properties of nanoparticles of

*Author for correspondence (mabdulkhadar@rediffmail.com) nickel ferrite have not been reported. The properties of nanoparticles are interesting due to the presence of very large and highly disordered grain boundaries. Because of the peculiarities of the grain boundaries nanomaterials exhibit unusual and/or enhanced dielectric properties.

In the present article, we report the preparation and study of microwave dielectric properties of consolidated nanoparticles of nickel ferrite having average grain sizes 10,20 and $23 \mathrm{~nm}$ in the $\mathrm{S}$ band frequency range (2$4 \mathrm{GHz}$ ). These properties are compared with those of sintered pellets of nanoparticles of nickel ferrite. The microwave properties like dielectric constant, dielectric loss and heating effect are investigated.

\section{Sample preparation and experimental procedure}

Nanoparticles of nickel ferrite were prepared by co-precipitation method using analar grade chemicals. Nickel nitrate, ferric nitrate, ammonium carbonate and sodium hydroxide were used as the starting materials. Ethylenedinitrinotetraacetic acid disodium salt (EDTA) was used as stabilizer. Aqueous solutions of ferric nitrate (10 ml, 0.5 molar) and nickel nitrate $(10 \mathrm{ml}, 0.5$ molar $)$ were added slowly to a mixture of $\mathrm{NaOH}(10 \mathrm{ml}$, 0.5 molar $),\left(\mathrm{NH}_{4}\right)_{2} \mathrm{CO}_{3}(10 \mathrm{ml}, 0.5$ molar $)$ and EDTA (10 ml, $0 \cdot 1$ molar) solutions kept under vigorous stirring. The stabilizer (EDTA) was used to prevent growth and agglomeration of the particles. The precipitate formed was separated and washed several times in distilled water to free it from ions and other impurities. Finally it was washed in acetone and dried at $75^{\circ} \mathrm{C}$ for $3 \mathrm{~h}$ to get the asprepared sample. 
The as-prepared sample was annealed at different temperatures to prepare the nanoparticles of nickel ferrite. The minimum temperature of annealing was selected after determining decomposition temperature of the as-prepared sample by TGA analysis. The differential plot of the TGA showed a peak at $262^{\circ} \mathrm{C}$, corresponding to the decomposition temperature of the as-prepared sample. Three samples of nickel ferrite nanoparticles were prepared by selecting different annealing temperatures above $262^{\circ} \mathrm{C}$. Sample codes assigned to the three samples along with the annealing temperatures, and duration of annealing are listed in table 1. X-ray diffraction (XRD) patterns of the samples were recorded using $\mathrm{CuK}_{\alpha}$ radiation.

To study the microwave dielectric properties, the samples NF1, NF2 and NF3 were compacted in the form of pellets of $13 \mathrm{~mm}$ diameter and about $1 \mathrm{~mm}$ thickness by applying a pressure of $\sim 0.4 \mathrm{GPa}$. A few pellets of the samples NF1 and NF3 were sintered at $1200^{\circ} \mathrm{C}$ for $12 \mathrm{~h}$ to get samples with sample codes NF1S and NF3S, respectively.

The experimental set up for measuring the dielectric properties in the microwave region consisted of a transmission type S-band rectangular cavity resonator, an HP 8714 ET network analyser and an interfacing computer (Mathew and Raveendranath 1993). The microwave properties of the five samples NF1, NF2, NF3, NF1S and NF3S were investigated at the $S$ band frequency range from 2.4-4 GHz. The cavity resonator was excited in the $\mathrm{TE}_{10 \mathrm{p}}$ mode and typical resonant frequency spectrum was obtained. Initially, the resonant frequency, $f_{0}$ and the corresponding quality factor, $Q_{0}$, of each resonant peak of the empty cavity were determined. Samples prepared in the form of pellets were then introduced into the cavity resonator through a non-radiating slot of the rectangular cavity. One of the resonant frequencies of the loaded cavity was selected and the position of the sample was adjusted for maximum perturbation (i.e. maximum shift of the resonant frequency towards the low frequency side with minimum amplitude for the peak). The new resonant frequency, $f_{\mathrm{s}}$ and 3-dB bandwidth $\left(\Delta f_{\mathrm{s}}\right)$ were determined and hence the quality factor, $Q_{\mathrm{s}}$, was calculated by the equation

$$
Q_{\mathrm{s}}=\frac{f_{\mathrm{s}}}{\Delta f_{\mathrm{s}}} .
$$

Table 1. Sample codes, annealing conditions and average grain size of nanoparticles of $\mathrm{NiFe}_{2} \mathrm{O}_{4}$.

\begin{tabular}{lccc}
\hline $\begin{array}{l}\text { Sample } \\
\text { code }\end{array}$ & $\begin{array}{c}\text { Annealing } \\
\text { temperature }\end{array}$ & Duration & Size (nm) \\
\hline NF1 & $275^{\circ} \mathrm{C}$ & $2 \mathrm{~h}$ & 10 \\
NF2 & $450^{\circ} \mathrm{C}$ & $2 \mathrm{~h}$ & 20 \\
NF3 & $600^{\circ} \mathrm{C}$ & $2 \mathrm{~h}$ & 23 \\
NF1S & Pellet of NF1 sintered at $1200^{\circ} \mathrm{C}$ for $12 \mathrm{~h}$ & \\
NF3S & Pellet of NF3 sintered at $1200^{\circ} \mathrm{C}$ for $12 \mathrm{~h}$ & \\
\hline
\end{tabular}

The procedure was repeated for other resonant frequencies.

\section{Results and discussion}

The XRD patterns of the samples are given in figure 1 . The peaks in the spectra agreed closely with the data in the ICDD file (card number 10-325) for $\mathrm{NiFe}_{2} \mathrm{O}_{4}$ indicating that the samples synthesized were nickel ferrite. The diffraction pattern of the samples showed broad peaks when compared with the bulk nickel ferrite. The average grain sizes of the particles were calculated from the full width at half maximum (FWHM) values of (311) reflection of the X-ray diffraction using Scherrer's equation (Klug and Alexander 1954). The calculated values of average grain sizes of the three samples are listed in table 1 . These samples of nanoparticles of nickel ferrite were employed to study the variation in microwave dielectric properties with the grain size.

The dielectric constant of the samples NF1, NF2, NF3 and of the sintered samples NF1S and NF3S over the microwave frequency range from $2.4-4 \mathrm{GHz}$ are shown in figure 2. The plots show that while the dielectric constant of each sample remains fairly constant over the frequency range of study, its value decreases with increase in grain size. The maximum value of dielectric constant, $10 \cdot 5$, is observed for the sample NF1 and minimum is 4.5 for the sample, NF3S.

The high value of dielectric constant of the sample NF1 may be explained on the basis of the structural changes associated with the nickel ferrite when the grain size is reduced to nanometer order. Nickel ferrite crystallizes into a cubic close-packed arrangement of oxygen ions. It belongs to the class of ferrites with an inverse spinel structure having structural formula, $\mathrm{Fe}^{3+}\left[\mathrm{Ni}^{2+} \mathrm{Fe}^{3+}\right] \mathrm{O}_{4}$ (Ponpandian et al 2002). The metal ions given in the square bracket are called octahedral (B site) ions and that outside the square bracket are called tetrahedral (A site) ions. The nickel ions $\left(\mathrm{Ni}^{2+}\right)$ together with half of the iron ions $\left(\mathrm{Fe}^{3+}\right)$ occupy the $\mathrm{B}$ site and the remaining half of the iron ions reside in A site. The presence of $\mathrm{Ni}^{2+}$ and $\mathrm{Ni}^{3+}$ ions gives rise to $p$-type carriers (holes) whereas $\mathrm{Fe}^{2+}$ and $\mathrm{Fe}^{3+}$ ions produce $n$-type carriers (electrons). Therefore, both electrons and holes that are present in the $\mathrm{B}$ sites are due to the presence of $\mathrm{Ni}$ and $\mathrm{Fe}$ ions. Since only iron ions are present in A sites, electrons are the carriers in A sites. The distance between the ions in A sites $(0.357 \mathrm{~nm})$ is larger than the distance between the ions in B site $(0.292 \mathrm{~nm})$ (Chinnasamy et al 2000). Also, the degree of covalency for the A site ions is higher than that of the B site ions. All the above factors result in a high activation energy for the A sites compared to the $\mathrm{B}$ sites. Hence, in ordinary nickel ferrite with an inverse spinel structure the electron movement in B sites dominates compared to that in A sites. 
Recently, it was reported that nickel ferrite deviates from its inverse spinel structure with the reduction in grain size (Ponpandian et al 2002). When the grain size of nickel ferrite is reduced to nanometer order, some percentage of nickel ions get shifted from B site to A site at the same time shifting an equal number of iron ions from A to B site. The percentage of iron ions in B site increases with the reduction in grain size. This shifting of

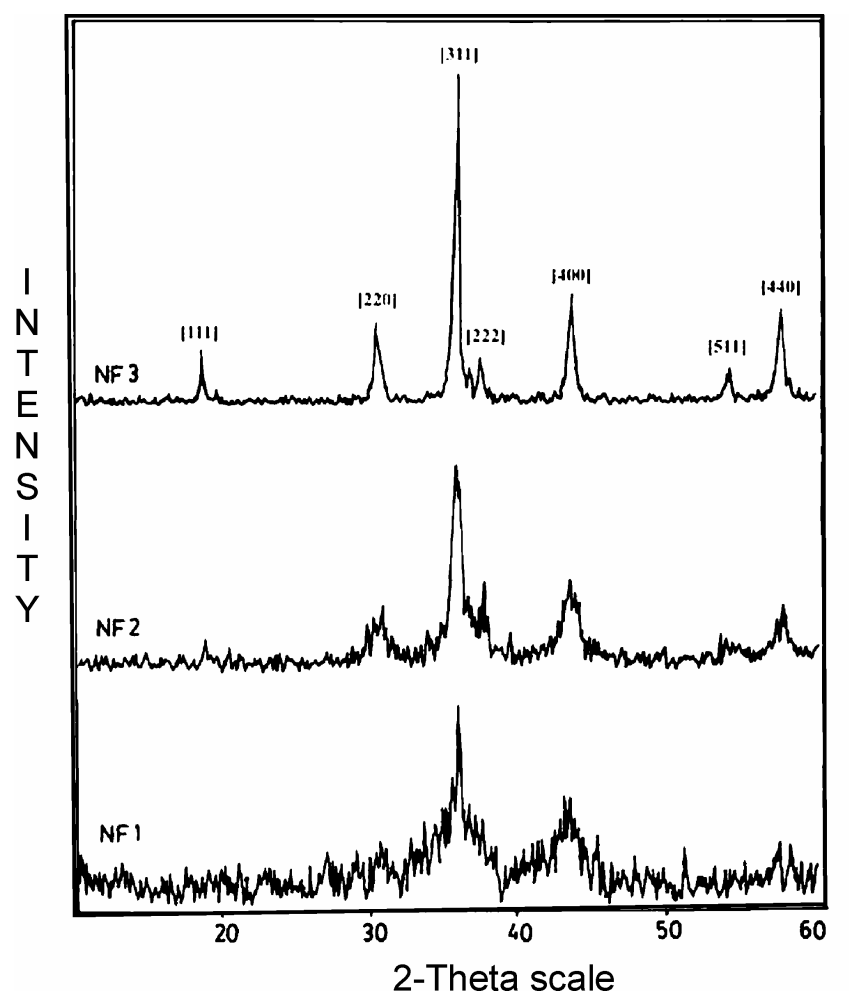

Figure 1. X-ray diffraction patterns of nanoparticles of nickel ferrite.

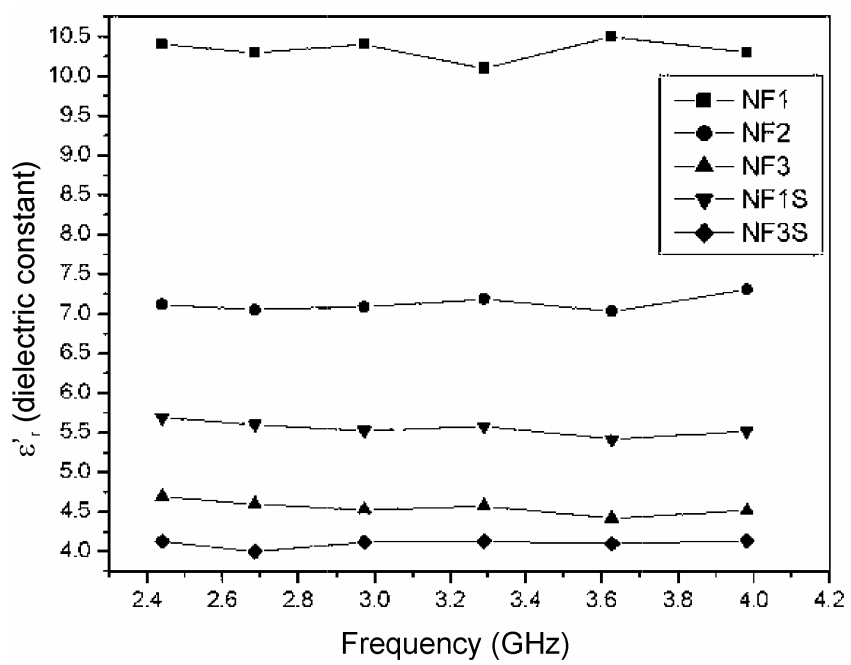

Figure 2. Variation of dielectric constant of nanostructured $\mathrm{NiFe}_{2} \mathrm{O}_{4}$ with frequency. ions causes changes in XRD pattern also (Sepelak et al 1998). As the percentage of iron ions in B site increases, the intensity of the (222) peak compared to that of the (220) peak increases. From figure 1, it is clear that the ratio of intensity of the (222) to (220) peaks of X-ray diffraction is high for the samples, NF1 and NF2, compared to that of the sample, NF3. This indicates that the structural changes of the samples, NF1 and NF2, arise from the shifting of ions between A and B sites. This shifting of ions is assumed to be prominent near the surface layers. Because the arrangement of ions in the core region of nanoparticles is identical with that in bulk crystals, while the altered structure due to the reduction in the size is confined to surface layers. As the size of a particle is reduced the surface to volume ratio increases and the number of iron ions in B sites increases. This results in crea-

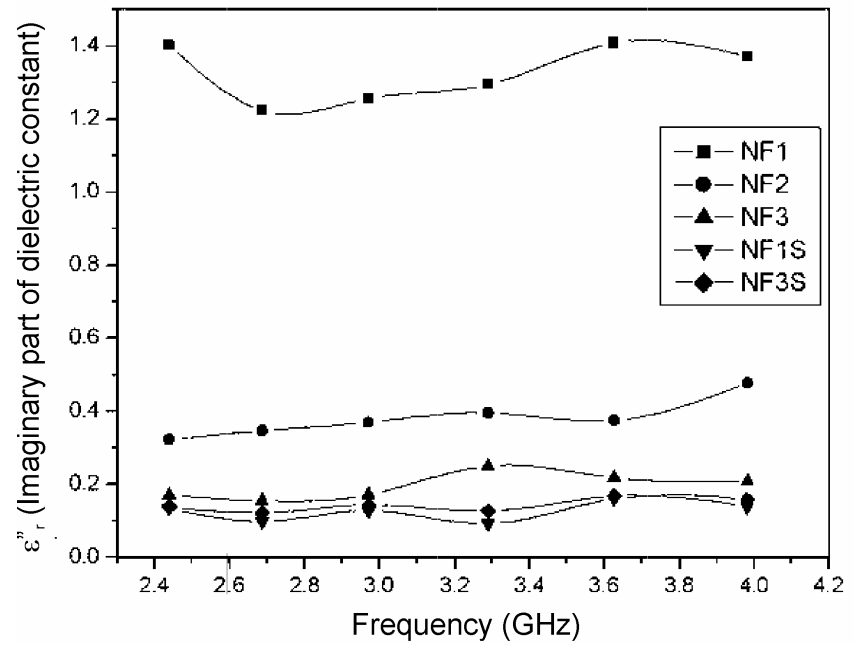

Figure 3. Variation of imaginary part of dielectric constant of nanostructured $\mathrm{NiFe}_{2} \mathrm{O}_{4}$ with frequency.

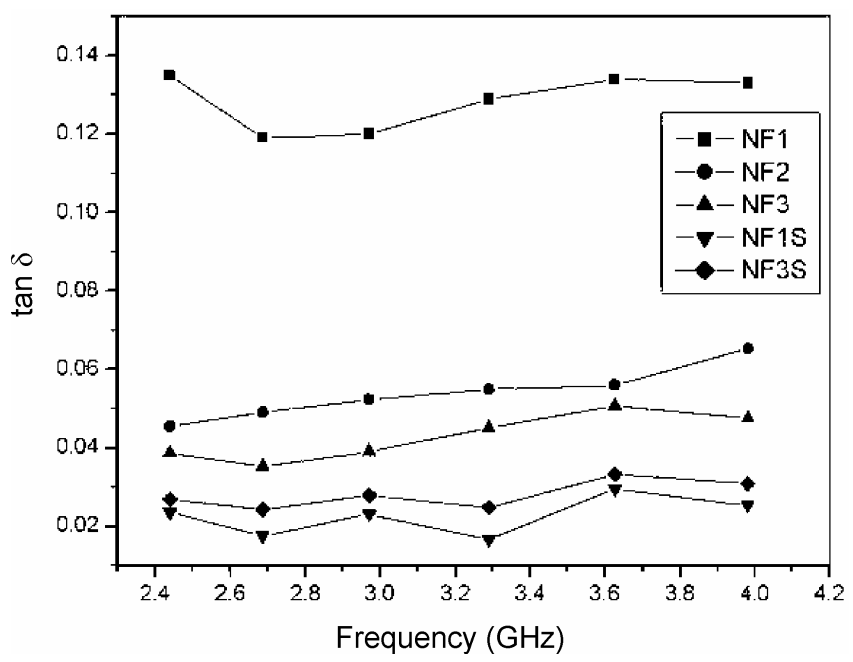

Figure 4. Variation of dielectric loss tangent of nanostructured $\mathrm{NiFe}_{2} \mathrm{O}_{4}$ with frequency. 


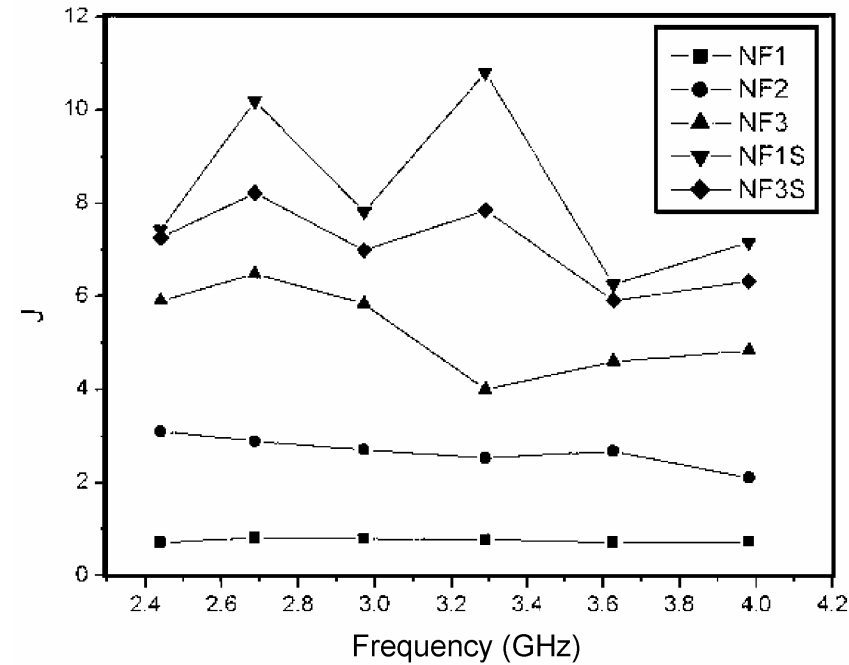

Figure 5. Variation of microwave heating coefficient of nanostructured $\mathrm{NiFe}_{2} \mathrm{O}_{4}$ with frequency.

tion of large number of $\mathrm{Fe}^{2+}$ ions since the activation energy in B site is comparatively lower than A site (Chinnasamy et al 2000). The increase in number of $\mathrm{Fe}^{2+}$ ions causes an increase in dielectric constant of nickel ferrite. This is because at microwave frequencies the dielectric constant of ferrites is related with the number of $\mathrm{Fe}^{2+}$ ions since they are easily polarized than $\mathrm{Fe}^{3+}$ ions (Verma et al 2003). Hence, it is concluded that the high value of dielectric constant of the sample, NF1, may be due to the large surface to volume ratio and the resulting increase in the number of $\mathrm{Fe}^{2+}$ ions in $\mathrm{B}$ sites.

Variation of the imaginary part of the dielectric constant $\left(\varepsilon_{\mathrm{r}}^{\prime \prime}\right)$ with frequency is shown in figure 3 . The maximum value of $\varepsilon_{\mathrm{r}}^{\prime \prime}$ is 1.4 at $2.4 \mathrm{GHz}$ for the sample, NF1. The value does not show a considerable variation with frequency. For the sintered samples, NF1S and $\mathrm{NF} 3 \mathrm{~S}, \varepsilon_{\mathrm{r}}$ " values are found to be one order of magnitude less than that of NF1 over the frequency range.

The dielectric loss tangent is defined as $\tan \delta=\varepsilon_{\mathrm{r}}^{\prime \prime} / \varepsilon_{\mathrm{r}}^{\prime}$. The variation of dielectric loss tangent of nanostructured $\mathrm{NiFe}_{2} \mathrm{O}_{4}$ with frequency is shown in figure 4 . The dielectric loss is a result of the lag in polarization in accordance with the applied electric field. Green et al (1964) reported the microwave properties of nickel ferrite particles of micron size range. It was observed that the $x$-band dielectric loss tangent of the fine grain (around $1 \mu \mathrm{m}$ ) sample is about $10^{-2}$, whereas the conventional samples $(30 \mu \mathrm{m})$ have a value $<10^{-3}$. The $\tan \delta$ for the sample NF 1 is of the order of $10^{-1}$, whereas the other samples have values of the order $10^{-2}$. The value decreases with the increase in grain size.

For the development of high frequency heating devices, materials with high $\tan \delta$ are employed. The microwave heating effect is expressed through a coefficient called the heating coefficient. It is defined as (Mathew et al 2003)

$$
J=\frac{1}{\varepsilon_{\mathrm{r}} \tan \delta} .
$$

The variation of microwave heating coefficient of nanostructured $\mathrm{NiFe}_{2} \mathrm{O}_{4}$ with frequency is shown in figure 5 . The microwave heating coefficient of the sample, NF1, is found to be uniform in the frequency range. The coefficient shows a size dependent variation. The sample, NF1, is having values of the coefficient much lower than the other three samples. The sintered samples have maximum values for the coefficient.

\section{Conclusions}

Nanostructured nickel ferrite samples were prepared through a chemical precipitation method followed by thermal processes. The microwave dielectric properties like dielectric constant, dielectric loss and microwave heating coefficient of nanostructured nickel ferrite samples of three different average grain sizes and those of two sintered samples were studied. The nanoparticles of nickel ferrite showed substantial variation in the values of all the above microwave parameters with average grain size.

\section{References}

Chinnasamy C N, Narayanasamy A, Ponpandian N, Chattopadhyay K, Gueralt H and Greneche J M 2000 J. Phys. Condens. Matter 127795

Giannakopoulou T, Kompotiatis L, Kontogeorgakos A and Kordas G 2002 J. Magn. Magn. Mater. 246360

Goldman A 1990 Modern ferrite technology (New York: Van Nostrand Reinhold) p. 71

Gopal Reddy C V, Manorama S V and Rao V J 1999 Sensors and Actuators B55 90

Gotic M, Czako-Nagy I, Popovic S and Music S 1998 Philos. Mag. 78193

Green J J, Waugh J S and Healy B J 1964 J. Appl. Phys. 351006

Han K C, Choi H D, Moon T J and Kim W S 1995 J. Mater. Sci. 303567

Hudson A S 1970 The Marconi Rev. 3321

Klug H P and Alexander L E 1954 X-ray powder diffraction procedure (New York: John Wiley) p. 504

Luo H Y, Yue Z X and Zhou J 2000 J. Magn. Magn. Mater. 210 104

Malinofski W W and Babbitt R W 1964 J. Appl. Phys. 351012

Mathew K T and Raveendranath U 1993 Microwave and Optical Tech. Lett. 6104

Mathew K T, Biju Kumar S, Anil Lonappan, Joe Jacob, Thomas Kurien, Jacob Samuel and Thommachan Xavier 2003 Mater. Chem. Phys. 79187

Meshram M R et al 2002 Bull. Mater. Sci. 25169

Nakamura T, Tsuoka T and Hatakayama K 1994 J. Magn. Magn. Mater. 138319 
Nicolas J 1980 Ferromagnetic materials (ed.) E P Wohlfarth (Amsterdam: North-Holland) p. 243

Ponpandian N, Balaya P and Narayanasamy A N 2002 J. Phys.: Condens. Matter 143221

Sepelak V, Buchal A, Tkacova K and Becker K D 1998 Mater. Sci. Forum 16862
Van Uitert L G 1956a J. Appl. Phys. 27723

Van Uitert L G 1956b Proc. IRE 441294

Verma A, Saxena A K and Dube D C 2003 J. Magn. Magn. Mater. 263228

Vladikova D, Yonchev H, Ilkov L and Karbanov S $1989 \mathrm{~J}$. Magn. Magn. Mater. 78420 